\title{
STABILITY OF INDIVIDUAL ELEMENTS UNDER ONE-PARAMETER SEMIGROUPS
}

\author{
CHARLES J. K. BATTY AND VŨ QUÔC PHÓNG
}

\begin{abstract}
Let $\{T(t): t \geq 0\}$ be a $C_{0}$-semigroup on a Banach space $X$ with generator $A$, and let $x \in X$. If $\sigma(A) \cap i \mathbf{R}$ is empty and $t \mapsto T(t) x$ is uniformly continuous, then $\|T(t) x\| \rightarrow 0$ as $t \rightarrow \infty$. If the semigroup is sun-reflexive, $\sigma(A) \cap i \mathbf{R}$ is countable, $P \sigma(A) \cap i \mathbf{R}$ is empty, and $t \mapsto T(t) x$ is uniformly weakly continuous, then $T(t) x \rightarrow 0$ weakly as $t \rightarrow \infty$. Questions of almost periodicity and of stabilization of contraction semigroups on Hilbert space are also discussed.
\end{abstract}

\section{INTRODUCTION}

Let $\mathscr{T}=\{T(t): t \geq 0\}$ be a bounded $C_{0}$-semigroup on a Banach space $X$, with generator $A$. The spectrum $\sigma(A)$ of $A$ is contained in the left halfplane $\{\lambda \in \mathbf{C}: \operatorname{Re} \lambda \leq 0\}$. Suppose that the peripheral spectrum, $\sigma(A) \cap i \mathbf{R}$, is countable, and the peripheral residual spectrum, $R \sigma(A) \cap i \mathbf{R}$, is empty. Then $\|T(t) x\| \rightarrow 0$ as $t \rightarrow \infty$, for each $x$ in $X$.

Two independent proofs have been given of this stability theorem $[1,13]$. In [1], an estimate on $\lim \sup _{t \rightarrow \infty}\|T(t) x\|$, for certain $x$ in $X$, was obtained by modifying a contour integral introduced by Korevaar [9] and Zagier [22] to obtain a simple proof of a Tauberian theorem for Laplace transforms (itself a special case of an old Tauberian theorem of Ingham [8]) and hence of the Prime Number Theorem. Then the estimate can be modified and extended iteratively to larger classes of vectors $x$, until the conclusion of strong convergence on $X$ is reached. If $\sigma(A) \cap i \mathbf{R}$ is empty, the proof is very simple and explicit; indeed, in this case, $\left\|T(t) A^{-1}\right\| \rightarrow 0$. In cases when $\sigma(A) \cap i \mathbf{R}$ has reasonably simple structure, the proof remains fairly explicit, but if the peripheral spectrum is complicated (while remaining countable), the proof does become obscure, the general case requiring transfinite induction.

The proof of the same stability theorem in [13] is by reductio ad absurdum. It deals with all cases, whatever the structure of $\sigma(A) \cap i \mathbf{R}$, in the same way, and therefore provides a succinct method for the general case. However, it is less elementary than [1] when $\sigma(A) \cap i \mathbf{R}$ has a simple structure.

Received by the editors August 31, 1989.

1980 Mathematics Subject Classification (1985 Revision). Primary 47D05; Secondary 47A10.

Key words and phrases. $C_{0}$-semigroup, stability, residual spectrum, sun-reflexive, stabilization, almost periodic. 
In [1] it was noted that stability of an individual element $x$ could sometimes be obtained by the same method, even if $\mathscr{T}$ is not bounded. If $\sigma(A) \subset\{\lambda \in$ $\mathrm{C}: \operatorname{Re} \lambda<0\}, x \in D(A)$, and $\|T(t) A x\|$ is bounded, then $\|T(t) x\| \rightarrow 0$. The present paper contains some further individual stability results. It is shown (Theorem 1) that $\|T(t) x\| \rightarrow 0$ provided that $A \sigma(A) \cap i \mathbf{R}$ is empty and $t \mapsto$ $T(t) x$ is uniformly continuous. A duality argument, combined with the method of [1], shows that $T(t) x \rightarrow 0$ weakly if $\mathscr{T}^{*}$ is strongly continuous, $\sigma(A) \cap i \mathbf{R}$ is countable, $R \sigma\left(A^{*}\right) \cap i \mathbf{R}$ is empty, and $T(t) x$ is bounded (Corollary 3 ), or if $\mathscr{T}$ is sun-reflexive, $\sigma(A) \cap i \mathbf{R}$ is countable, $P \sigma(A) \cap i \mathbf{R}$ is empty, and $t \mapsto T(t) x$ is uniformly weakly continuous (Corollary 5 ). In $\S 3$, two examples are given to show that these results are close to being optimal.

In $\S 4$, we show how our methods can be used to prove that a bounded $C_{0}$ semigroup $\mathscr{T}$ is almost periodic if $\sigma(A) \cap i \mathbf{R}$ is countable and $\operatorname{Ran}(A-i \mu)+$ $\operatorname{ker}(A-i \mu)$ is dense in $X$ for each real $\mu$ [19]. In $\S 5$, we show how almost periodicity can be used to give an alternative proof, and slight improvements, of results of Korobov and Sklyar [10] concerning problems of stabilization of semigroups on Hilbert space arising in control theory.

Throughout the paper, $\mathscr{T}=\{T(t): t \geq 0\}$ will be a $C_{0}$-semigroup on a Banach space $X, A$ will be its generator, $\rho(A)$ the resolvent set of $A$, and $\sigma(A), P \sigma(A), A \sigma(A)$, and $R \sigma(A)$ the spectrum, point spectrum, approximate point spectrum, and residual spectrum of $A$, respectively. Thus,

$$
R \sigma(A)=\{\lambda \in \mathbf{C}: \text { the range of } \lambda-A \text { is not dense in } X\}=P \sigma\left(A^{*}\right) .
$$

We are grateful to W. Arendt, Yu. I. Lyubich and G. M. Sklyar for valuable comments and suggestions about this work. The paper was completed during a visit to Oxford by the second author.

\section{Stability THEOREMS}

In this section, we give two individual stability theorems, one giving strong stability, and the other leading to weak stability. It should be noted that, in neither theorem, is it assumed that the spectrum $\sigma(A)$ of the generator $A$ is contained in the left half-plane. In the first theorem (and in Corollary 4 ) it is not assumed that $T(t) x$ is bounded (although this follows from the conclusion of the theorem).

Theorem 1. Let $\{T(t): t \geq 0\}$ be a $C_{0}$-semigroup on a Banach space $X$, with generator $A$, and suppose that $A \sigma(A) \cap i \mathbf{R}$ is empty. Let $x \in X$, and suppose that $t \mapsto T(t) x$ is uniformly continuous. Then $\|T(t) x\| \rightarrow 0$ as $t \rightarrow \infty$.

Proof. Let $Y$ be the closed linear span of $\{T(t) x: t \geq 0\}$ in $X$, and $A_{0}$ be the part of $A$ in $Y$, so that $D\left(A_{0}\right)=D(A) \cap Y, A_{0} y=A y \quad\left(y \in D\left(A_{0}\right)\right)$. Let $\alpha(t)=T(t) x$. Since $\alpha$ is uniformly continuous, there are constants $c, d$ such that $\|\alpha(t)\| \leq c t+d$ for all $t>0$. Hence

$$
\int_{0}^{\infty} e^{-i t}\|\alpha(t)\| d t<\infty
$$


for all $\lambda>0$. Let

$$
\begin{gathered}
g(z)=z \int_{0}^{\infty} e^{-z s} \alpha(s) d s \quad(\operatorname{Re} z>0), \\
g_{t}(z)=e^{-z t} \alpha(t)+z \int_{0}^{t} e^{-z s} \alpha(s) d s \quad(z \in \mathbf{C}, t>0) .
\end{gathered}
$$

By a standard argument, it can be seen that $g(z) \in D\left(A_{0}\right)$ and $A(g(z))=$ $A_{0}(g(z))=z g(z)-z x$. Since $0 \notin A \sigma(A)$,

$$
0<\liminf _{\substack{z \rightarrow 0 \\ \operatorname{Re} z>0}} \frac{\|A(g(z))\|}{\|g(z)\|}=\liminf _{\substack{z \rightarrow 0 \\ \operatorname{Re} z>0}} \frac{|z|\|x\|}{\|g(z)\|}
$$

so $\|g(z)\| /|z|$ is bounded, as $z \rightarrow 0, \operatorname{Re} z>0$. Hence $-z^{-1} A_{0}(g(z)) \rightarrow x$, so $x$ is in the closure of the range of $A_{0}$. Since this range is $\mathscr{T}$-invariant, it is dense in $Y$. Thus $0 \notin \sigma\left(A_{0}\right)$. A similar argument shows that $i \mu \notin \sigma\left(A_{0}\right)$ $(\mu \in \mathbf{R})$.

Now the function $g(z)$ is analytic for $\operatorname{Re} z>0$ and

$$
g(z)=z\left(z-A_{0}\right)^{-1} x \quad\left(x \in \rho\left(A_{0}\right), \operatorname{Re} z>0\right) .
$$

Thus $g$ extends to an analytic function in $\rho\left(A_{0}\right) \cup\{z: \operatorname{Re} z>0\}$, which is an open set containing the imaginary axis.

Since $\alpha$ is uniformly continuous,

$$
\lim _{\delta \downarrow 0} \limsup \sup _{t \rightarrow \infty \leq s \leq t}|\alpha(s)-\alpha(t)|=0 .
$$

It therefore follows from [2, Theorem 2.3] that $g_{t}(0) \rightarrow g(0)$ as $t \rightarrow \infty$, so that $\|\alpha(t)\| \rightarrow 0$.

Remarks. 1. The reader should be aware that the essence of the proof of Theorem 1 is to apply an estimate of Korevaar [9] and Zagier [22], obtained from a simple contour integral, to show that $\left\|T(t) x_{\delta}\right\| \rightarrow 0$, where $x_{\delta}:=$ $\delta^{-1} \int_{0}^{\delta} T(t) x d t$ (so that $T(t) x_{\delta}$ is bounded), and then to use the fact that $T(t) x_{\delta} \rightarrow T(t) x$, uniformly in $t$, as $\delta \downarrow 0$.

2. Theorem 1 remains true if the assumption that $A \sigma(A) \cap i \mathbf{R}$ is empty is replaced by the assumption that the function $g(z)$ defined by $(*)$ has an analytic extension across the imaginary axis.

The formulation of the next result requires the notion of the sun-dual $\mathscr{T}^{\odot}$ of $\mathscr{T}[7$, Chapter $14 ; 3, \S 3.4]$. Let

$$
X^{\odot}=\left\{\phi \in X^{*}:\left\|T^{*}(t) \phi-\phi\right\| \rightarrow 0 \text { as } t \downarrow 0\right\} .
$$

Then $X^{\odot}$ is a norm-closed $\mathscr{T}^{*}$-invariant subspace of $X^{*}$; moreover, $X^{\odot}$ is the norm-closure of $D\left(A^{*}\right)$. Let $T^{\odot}(t)=T^{*}(t) \mid X^{\odot}$. Then $\mathscr{T}^{\odot}$ is a $C_{0}$-semigroup on $X^{\odot}$, and its generator $A^{\odot}$ is given by

$$
D\left(A^{\odot}\right)=\left\{\phi \in D\left(A^{*}\right): A^{*} \phi \in X^{\odot}\right\}, \quad A^{\odot}=A^{*} \mid D\left(A^{\odot}\right) .
$$


The following spectral properties hold:

$$
\begin{gathered}
\sigma(A)=\sigma\left(A^{*}\right)=\sigma\left(A^{\odot}\right), \\
R \sigma(A)=P \sigma\left(A^{*}\right)=P \sigma\left(A^{\odot}\right), \\
R \sigma\left(A^{\odot}\right) \subseteq R \sigma\left(A^{*}\right) .
\end{gathered}
$$

Theorem 2. Let $\{T(t): t \geq 0\}$ be a $C_{0}$-semigroup on a Banach space $X$ with generator $A$, and suppose that $\sigma(A) \cap i \mathbf{R}$ is countable and $R \sigma\left(A^{\odot}\right) \cap i \mathbf{R}$ is empty. Let $x \in X$ and suppose that $\{T(t) x: t \geq 0\}$ is bounded. Then $\phi(T(t) x) \rightarrow 0$ as $t \rightarrow \infty$, for any $\phi$ in $X^{\odot}$. Hence $T(t)(\lambda-A)^{-1} x \rightarrow 0$ weakly as $t \rightarrow \infty$, for any $\lambda$ in $\rho(A)$.

Proof. For $\phi$ in $X^{\odot}$, let

$$
f_{\phi}(t)=\left(\hat{x} \circ T^{\odot}(t)\right) \phi=\left(T^{\odot}(t) \phi\right)(x)=\phi(T(t) x) .
$$

Then $f_{\phi}$ is a bounded continuous function, with Laplace transform

$$
g_{\phi}(z)=\int_{0}^{\infty} e^{-z t} f_{\phi}(t) d t .
$$

This is defined and analytic in the region $\operatorname{Re} z>0$. Moreover, if $z \in \rho(A)$, then

$$
g_{\phi}(z)=-\int_{0}^{\infty} \frac{d}{d t}\left(e^{-z t} \phi\left(T(t)(z-A)^{-1} x\right)\right) d t=\phi\left((z-A)^{-1} x\right) .
$$

Thus $g_{\phi}$ extends analytically to $\rho(A) \cup\{z: \operatorname{Re} z>0\}$.

Now the argument of $[1, \S 3]$ is applicable. (In the inductive hypothesis, the quantities $\left\|T(t) A^{-1} U x\right\|,\|U x\|,\left\|U_{j} x\right\|$ are replaced by $\left|\phi\left(T(t) A^{-1} U x\right)\right|$, $\left\|U^{*} \phi\right\|,\left\|U_{j}^{*} \phi\right\|$ respectively, and $M$ now represents $\left.\sup _{t>0}\|T(t) x\|.\right)$ The conclusion is that $\phi(T(t) x) \rightarrow 0$ for each $\phi$ in $X^{\odot}$. In particular, for $\psi$ in $X^{*},\left(\lambda-A^{*}\right)^{-1} \psi \in D\left(A^{*}\right) \subseteq X^{\odot}$, so

$$
\psi\left(T(t)(\lambda-A)^{-1} x\right)=\left(\left(\lambda-A^{*}\right)^{-1} \psi\right)(T(t) x) \rightarrow 0 .
$$

Corollary 3. Suppose that $\mathscr{T}^{*}$ is strongly continuous, $\sigma(A) \cap i \mathbf{R}$ is countable, and $R \sigma\left(A^{*}\right) \cap i \mathbf{R}$ is empty. Then $T(t) x \rightarrow 0$ weakly for each $x$ such that $\sup _{t>0}\|T(t) x\|<\infty$. Furthermore, there is a net $\left(T_{\alpha}\right)$ in the convex hull of $\{T(t): t \geq 0\}$ such that $\left\|T_{\alpha} x\right\| \rightarrow 0$ for all such $x$.

Proof. It is immediate from Theorem 2 that $T(t) x \rightarrow 0$ weakly. The final statement is a standard Hahn-Banach argument.

In the situation of Corollary 3 , it is not necessarily true that

$$
\left\|\frac{1}{t} \int_{0}^{t} T(s) x d s\right\| \rightarrow 0 \quad \text { as } t \rightarrow \infty
$$

(see Example 7). 
Corollary 4. Suppose that $\sigma(A) \cap i \mathbf{R}$ is countable and $R \sigma\left(A^{\odot}\right) \cap i \mathbf{R}$ is empty. Let $x \in X$ and suppose that $t \mapsto e^{-i \mu t} T(t) x$ is uniformly weakly continuous for some i $\mu$ in $\rho(A) \cap i \mathbf{R}$. Then $T(t) x \rightarrow 0$ weakly as $t \rightarrow \infty$.

Remark. A posteriori, we know that $T(t) x$ is bounded. Then the condition of uniform weak continuity is independent of $\mu$.

Proof. Replacing $T(t)$ by $e^{-i \mu t} T(t)$, we may assume that $\mu=0$ and $0 \in \rho(A)$. For $\delta>0$, let

$$
x_{\delta}=\frac{1}{\delta} \int_{0}^{\delta} T(t) x d t, \quad y_{\delta}=A x_{\delta}=\frac{1}{\delta}(T(\delta) x-x) .
$$

Then $\left\{T(t) y_{\delta}: t \geq 0\right\}$ is weakly bounded, hence bounded. It follows from Theorem 2 that $\phi\left(T(t) A x_{\delta}\right) \rightarrow 0$ for any $\phi$ in $X^{\odot}$. For $\psi$ in $D\left(A^{*}\right)$, we may put $\phi=A^{*-1} \psi \in D\left(A^{*}\right) \subseteq X^{\odot}$ and deduce that $\psi\left(T(t) x_{\delta}\right) \rightarrow 0$ as $t \rightarrow \infty$. But, as $\delta \downarrow 0, \psi\left(T(t) x_{\delta}\right) \rightarrow \psi(T(t) x)$ uniformly in $t$, by uniform weak continuity of $T(t) x$, so $\psi(T(t) x) \rightarrow 0$ as $t \rightarrow \infty$.

The construction of the sun-dual may be repeated to form $\left(X^{\odot \odot}, \mathscr{T}^{\odot \odot}\right)$ as the sun-dual of $\left(X^{\odot}, \mathscr{T}^{\odot}\right)$. There is a canonical (nonisometric) embedding $x \mapsto \hat{x}$ of $X$ in $X^{\odot \odot}$ given by

$$
\hat{x}(\phi)=\phi(x) \quad\left(\phi \in X^{\odot}\right) .
$$

Since $T^{\odot \odot}(t) \hat{x}=(T(t) x)^{\wedge}$, we may identify $(X, \mathscr{T})$ with a subspace of $\left(X^{\odot \odot}, \mathscr{T}^{\odot \odot}\right)$. If $X^{\odot \odot}=X, \mathscr{T}$ is said to be sun-reflexive. De Pagter [5] has shown that $\mathscr{T}$ is sun-reflexive if and only if the resolvents $(\lambda-A)^{-1}$ are weakly compact operators on $X$, for $\lambda \in \rho(A)$. If $X$ is reflexive, then any $C_{0}$-semigroup on $X$ is sun-reflexive. The translation semigroup on $C(\mathbf{T})$ is sun-reflexive, but the translation semigroup on $C_{0}(\mathbf{R})$ is not.

Corollary 5. Suppose that $\mathscr{T}$ is sun-reflexive, $\sigma(A) \cap i \mathbf{R}$ is countable, $P \sigma(A) \cap i \mathbf{R}$ is empty, and let $x \in X$. If $\{T(t) x: t \geq 0\}$ is bounded, then $\phi(T(t) x) \rightarrow 0$ as $t \rightarrow \infty$ for any $\phi$ in $X^{\odot}$. If $t \mapsto e^{-i \mu t} T(t) x$ is uniformly weakly continuous for some i $\mu$ in $\rho(A) \cap i \mathbf{R}$, then $T(t) x \rightarrow 0$ weakly.

Proof. Since $\mathscr{T}$ is sun-reflexive,

$$
R \sigma\left(A^{\odot}\right)=P \sigma\left(A^{\odot *}\right)=P \sigma\left(A^{\odot \odot}\right)=P \sigma(A),
$$

so Theorem 2 and Corollary 4 are applicable.

\section{EXAMPLES}

In this section, we give examples to show that the previous results are close to being optimal. The first example includes Example $2.5 \mathrm{~b}$ ) of [1], but it also shows that similar effects can occur in reflexive spaces, and that, in nonreflexive cases, Theorem 2 and its corollaries cannot be strengthened to give weak convergence under more general conditions. 
Example 6. Let $X=l^{p},(1 \leq p<\infty)$ or $X=c_{0}$. Define a $C_{0}$-semigroup on $X$ by

$$
\begin{aligned}
(T(t) x)_{2 n-1} & =e^{i n t-t / n^{2}}\left(x_{2 n-1}+t x_{2 n}\right), \\
(T(t) x)_{2 n} & =e^{i n t-t / n^{2}} x_{2 n} .
\end{aligned}
$$

The generator $A$ is given by

$$
\begin{aligned}
D(A) & =\left\{x \in X:\left(n x_{n}\right) \in X\right\}, \\
(A x)_{2 n-1} & =x_{2 n}+\left(\text { in }-1 / n^{2}\right) x_{2 n-1}, \\
(A x)_{2 n} & =\left(\text { in }-1 / n^{2}\right) x_{2 n}, \\
\sigma(A) & =\left\{\text { in }-1 / n^{2}: n \geq 1\right\} .
\end{aligned}
$$

We identify $X^{*}$ with $l^{q}$ where $1 / p+1 / q=1\left(q=1\right.$ if $\left.X=c_{0}\right)$. Then

$$
\begin{aligned}
& X^{\odot}= \begin{cases}l^{q} & \left(X=l^{p}, 1<p<\infty\right), \\
c_{0} & \left(X=l^{1}\right), \\
l^{1} & \left(X=c_{0}\right),\end{cases} \\
& \left(T^{\odot}(t) x\right)_{2 n-1}=e^{i n t-t / n^{2}} x_{2 n-1}, \\
& \left(T^{\odot}(t) x\right)_{2 n}=e^{i n t-t / n^{2}}\left(x_{2 n}+t x_{2 n-1}\right) .
\end{aligned}
$$

The semigroup is sun-reflexive.

Now define $y \in X$ by

$$
y_{2 n-1}=0, \quad y_{2 n}=n^{-(2 p+1) / p} \quad\left(y_{2 n}=n^{-2} \text { if } X=c_{0}\right) .
$$

Then (if $X=l^{p}$ ),

$$
\begin{aligned}
(T(t) y)_{2 n-1} & =\frac{t}{n^{(2 p+1) / p}} e^{i n t-t / n^{2}}, \\
(T(t) y)_{2 n} & =\frac{1}{n^{(2 p+1) / p}} e^{i n t-t / n^{2}} .
\end{aligned}
$$

A simple argument involving Riemann sums of $s^{-(2 p+1)} e^{-p / s^{2}}$ shows that, as $t \rightarrow \infty$,

$$
\begin{aligned}
\|T(t) y\|^{p}=\sum_{n=1}^{\infty} \frac{1+t^{p}}{n^{2 p+1}} e^{-p t / n^{2}} & \rightarrow \int_{0}^{\infty} s^{-(2 p+1)} e^{-p / s^{2}} d s \\
& =\frac{1}{2} \int_{0}^{\infty} u^{p-1} e^{-p u} d u=\frac{(p-1) !}{2 p^{p}}
\end{aligned}
$$

If $X=c_{0}$, then $\|T(t) y\| \rightarrow e^{-1}$. In each case, $\|T(t) y\|$ converges to a nonzero limit. Thus, in Theorem 1, it is not possible to replace the assumption of uniform continuity by boundedness, even in reflexive spaces. 
If $X=l^{p},(1<p<\infty)$ or $X=c_{0}$, then $X^{\odot}=X^{*}$ and it may be seen by direct calculation, or from Corollary 3 , that $T(t) x \rightarrow 0$ weakly whenever $T(t) x$ is bounded. However, if $p=1$, then $X^{*}=l^{\infty}, X^{\odot}=c_{0}$. If we put $\phi(x)=\sum_{1}^{\infty} x_{n},\left(x \in l^{1}\right)$, then, as $r \rightarrow \infty$,

$$
\begin{aligned}
\phi(T(2 \pi r) y) & =\|T(2 \pi r) y\|=\sum_{n=1}^{\infty}\left(\frac{1+2 \pi r}{n^{3}}\right) e^{-2 \pi r / n^{2}} \\
& \rightarrow 2 \pi \int_{0}^{\infty} \frac{1}{s^{3}} e^{-2 \pi / s^{2}} d s=\frac{1}{2}
\end{aligned}
$$

so $T(t) y$ does not converge weakly to 0 , even though $\sigma(A) \subset\{\lambda \in \mathrm{C}: \operatorname{Re} \lambda<$ $0\}, y \in D(A)$, and $T(t) y$ is bounded. This shows that Theorem 2 and its corollaries cannot be strengthened to give weak convergence under various more general conditions. Moreover, if we put

$$
f(t)=\phi(T(t) A y)=\sum_{n=1}^{\infty}\left\{1+(1+t)\left(i n-\frac{1}{n^{2}}\right)\right\} \frac{e^{i n t-t / n^{2}}}{n^{3}},
$$

we find that

$$
\begin{gathered}
|f(t)| \leq\|T(t)\|\|A y\|=\|A y\|(1+t), \\
g(z)=\int_{0}^{\infty} f(t) e^{-t z} d t=\phi\left((z-A)^{-1} A y\right),
\end{gathered}
$$

so the singular set of $g$ is empty, but

$$
\left|\int_{0}^{t} f(s) d s-g(0)\right|=|\phi(T(t) y)|,
$$

which does not tend to 0 as $t \rightarrow \infty$. This gives an example of a scalar-valued function $f$ to show that the assumption of boundedness of $f$ cannot be relaxed in even the simplest cases of Ingham's type of Tauberian theorem $[8,9,22 ; 1$, Theorem 4.1].

The second example shows that Theorem 2 and its corollaries cannot be improved to give strong convergence, except in circumstances covered by [1, Theorem 3.1] or Theorem 1. It also answers negatively a question raised in [1]. Note that the semigroup in this example is norm-continuous.

Example 7. Let $X=l^{p},(1<p<\infty)$ or $X=c_{0}$. Define a norm-continuous semigroup on $X$ by

$$
(T(t) x)_{2 n-1}=e^{-t / n}\left(x_{2 n-1}+t x_{2 n}\right), \quad(T(t) x)_{2 n}=e^{-t / n} x_{2 n} .
$$

The generator $A$ is given by

$$
\begin{gathered}
(A x)_{2 n-1}=x_{2 n}-\frac{1}{n} x_{2 n-1}, \quad(A x)_{2 n}=-\frac{1}{n} x_{2 n}, \\
\sigma(A)=\left\{-\frac{1}{n}: n \geq 1\right\} \cup\{0\}, \quad 0 \notin R \sigma(A) \cup R \sigma\left(A^{\odot}\right) .
\end{gathered}
$$

It may be seen by direct calculation, or from Corollary 3 , that if $T(t) x$ is bounded, then $T(t) x \rightarrow 0$ weakly as $t \rightarrow \infty$. 
Define $y$ in $X$ by

$$
y_{2 n-1}=0, \quad y_{2 n}=n^{-(p+1) / p} \quad\left(y_{2 n}=n^{-1} \text { if } X=c_{0}\right) .
$$

Now, as $t \rightarrow \infty$,

$$
\begin{gathered}
\|T(t) y\|=\left\{\sum_{n=1}^{\infty} \frac{\left(1+t^{p}\right) e^{-p t / n}}{n^{p+1}}\right\}^{1 / p} \rightarrow \frac{((p-1) !)^{1 / p}}{p} \quad\left(X=l^{p}\right), \\
\|T(t) y\| \rightarrow e^{-1} \quad\left(X=c_{0}\right) .
\end{gathered}
$$

Hence $t \mapsto\|T(t) y\|$ is bounded. Since $A$ is bounded, it follows that $t \mapsto$ $\|T(t) A y\|$ is bounded and $t \mapsto T(t) y$ is uniformly continuous. This shows that the question raised in [1, Remark 3.4] has a negative answer. Moreover, Theorem 2 cannot be improved to give strong convergence.

Note further that

$$
\begin{gathered}
\frac{1}{t} \int_{0}^{t}(T(s) y)_{2 n-1} d s=n^{-1 / p}\left\{\frac{n}{t}\left(1-e^{-t / n}\right)-e^{-t / n}\right\}, \\
\frac{1}{t} \int_{0}^{t}(T(s) y)_{2 n} d s=n^{-1 / p} \frac{\left(1-e^{-t / n}\right)}{t} .
\end{gathered}
$$

Thus, if $X=l^{p}$,

$$
\begin{gathered}
\left\|\frac{1}{t} \int_{0}^{t} T(s) y d s\right\|^{p}=\sum_{n=1}^{\infty} n^{-1}\left[\left\{\frac{n}{t}\left(1-e^{-t / n}\right)-e^{-t / n}\right\}^{p}+\left(\frac{1-e^{-t / n}}{t}\right)^{p}\right] \\
\rightarrow \int_{0}^{\infty} \frac{1}{s}\left(s\left(1-e^{-1 / s}\right)-e^{-1 / s}\right)^{p} d s>0 .
\end{gathered}
$$

If $X=c_{0}$, then

$$
\left\|\frac{1}{t} \int_{0}^{t} T(s) y d s\right\| \rightarrow \sup _{s>0}\left|s\left(1-e^{-1 / s}\right)-e^{-1 / s}\right|>0 .
$$

Thus, a mean ergodic theorem does not apply to $y$.

Finally, define $z$ in $X$ by

$$
\begin{aligned}
z_{2 r^{2}} & =1 / r \quad(r \geq 1), \\
z_{n} & =0 \quad \text { otherwise }
\end{aligned}
$$

If $s \geq t$,

$$
\begin{aligned}
\left|(T(s) z)_{2 r^{2}-1}-(T(t) z)_{2 r^{2}-1}\right| & =\frac{1}{r}\left|s e^{-s / r^{2}}-t e^{-t / r^{2}}\right| \leq \frac{s-t}{r}, \\
\left|(T(s) z)_{2 r^{2}}-(T(t) z)_{2 r^{2}}\right| & =\frac{1}{r}\left(e^{-t / r^{2}}-e^{-s / r^{2}}\right) \leq \frac{s-t}{r^{3}} \leq \frac{s-t}{r} .
\end{aligned}
$$

Hence

$$
\begin{aligned}
& \|T(s) z-T(t) z\| \leq(s-t)\left(\sum_{r=1}^{\infty} r^{-p}\right)^{1 / p} \quad\left(X=l^{p}\right), \\
& \|T(s) z-T(t) z\| \leq s-t \quad\left(X=c_{0}\right) .
\end{aligned}
$$


Thus $t \mapsto T(t) z$ is uniformly continuous. Nevertheless,

$$
\left\|T\left(r^{2}\right) z\right\| \geq\left(T\left(r^{2}\right) z\right)_{2 r^{2}-1}=r e^{-1} .
$$

This shows that, in Corollary 4, it is not possible to assume that $t \mapsto e^{-i \mu t} T(t) x$ is uniformly weakly continuous for some $i \mu$ in $\sigma(A) \cap i \mathbf{R}$.

\section{Almost PERIODIC SEMIGROUPS}

A bounded semigroup $\mathscr{T}$ is said to be almost periodic if each orbit $\{T(t) x$ : $t \geq 0\}$ is relatively compact. By the splitting theorem of Jacobs, de Leeuw, and Glicksberg [4; 11, Theorem 4.4, p. 105], if $\mathscr{T}$ is almost periodic, then

$$
X=X_{s}(\mathscr{T}) \oplus X_{b}(\mathscr{T}),
$$

where $X_{s}(\mathscr{T})=\{x \in X:\|T(t) x\| \rightarrow 0\}$ and $X_{b}(\mathscr{T})$ is the closed linear span of $\{x \in D(A): A x=i \mu x$ for some $\mu \in \mathbf{R}\}$. Moreover, $\mathscr{T} \mid X_{b}(\mathscr{T})$ extends to a bounded $C_{0}$-group on $X_{b}(\mathscr{T})$.

The following theorem has been obtained in [19] by a method similar to the stability theorem proved in [13]. We outline an alternative proof similar to [1].

Theorem 8. Let $\mathscr{T}$ be a bounded $C_{0}$-semigroup on a Banach space $X$, with generator $A$. Suppose that $\sigma(A) \cap i \mathbf{R}$ is countable. Then $\mathscr{T}$ is almost periodic if and only if, for each real $\mu, \operatorname{Ran}(A-i \mu)+\operatorname{ker}(A-i \mu)$ is dense in $X$.

Proof. Suppose that $\mathscr{T}$ is almost periodic, $f \in X^{*}$, and $f$ vanishes on $\operatorname{Ran}(A-i \mu)+\operatorname{ker}(A-i \mu)$. Then $A^{*} x=i \mu f$, so $T(t)^{*} f=e^{i \mu t} f$. If $x \in X_{s}(\mathscr{T})$, then

$$
e^{i \mu t} f(x)=f(T(t) x) \rightarrow 0,
$$

so $f(x)=0$. If $x \in \operatorname{ker}(A-i \mu)$, then $f(x)=0$ by assumption. If $x \in$ $\operatorname{ker}(A-i \lambda)$, where $\lambda \neq \mu$, then

$$
e^{i \mu t} f(x)=f(T(t) x)=e^{i \lambda t} f(x),
$$

so $f(x)=0$. Thus $f=0$, by the splitting theorem.

Conversely, suppose that, for each $\mu, \operatorname{Ran}(A-i \mu)+\operatorname{ker}(A-i \mu)$ is dense in $X$. Then a routine variation of a spectral mapping theorem [14, p. 85] shows that, for each $t>0, \operatorname{Ran}(T(t)-1)+\operatorname{ker}(T(t)-1)$ is dense in $X$. Suppose, without loss of generality, that $0 \in \rho(A)$. Now, in the notation of [1, p. 843], consider the following inductive hypothesis (where $\alpha$ is an ordinal):

If $E_{\alpha}$ is covered by disjoint intervals $\left(\eta_{j}-\varepsilon_{j}, \eta_{j}+\varepsilon_{j}\right) \quad(j=1, \ldots, n)$, where $\eta_{j} \in E_{\alpha},\left|\eta_{j}\right|-\varepsilon_{j}>0, R-\left|\eta_{j}\right|-\varepsilon_{j}>0$ and $\eta_{j} \pm \varepsilon_{j} \notin E \quad(j=1, \ldots, n)$, then $\left\{T(t) A^{-1} U x: t \geq 0\right\}$ has an $\varepsilon$-net whenever

$$
\varepsilon>\frac{2 M\|U x\|}{R} \prod_{j=1}^{n} \alpha_{j}+48 \pi M \sum_{j=1}^{n}\left\|U_{j} x\right\|\left|\eta_{j}\right| \varepsilon_{j}\left(\left|\eta_{j}\right|-\varepsilon_{j}\right)^{-1}\left(\eta_{j}^{2}-\varepsilon_{j}^{2}\right)^{-1} \prod_{\substack{k=1 \\ k \neq j}}^{n} \beta_{j k} .
$$


If $\alpha=0$, this follows immediately from the estimate, obtained in [1] by contour integration, on $\lim \sup _{t \rightarrow \infty}\left\|T(t) A^{-1} U x\right\|$. The inductive step for a nonlimit ordinal is carried out using the density of $\operatorname{Ran}(T(t)-1)+\operatorname{ker}(T(t)-1)$. Eventually when $E_{\alpha}$ is empty, we find that $\left\{T(t) A^{-1} x: t \geq 0\right\}$ has an $\varepsilon$-net whenever $\varepsilon>0$. Since $\mathscr{T}$ is bounded and $\operatorname{Ran}\left(A^{-1}\right)=D(A)$ is dense in $X$, it follows that $\{T(t) x: t \geq 0\}$ has an $\varepsilon$-net whenever $\varepsilon>0$, and is therefore relatively compact.

Remark. Suppose that $\mathscr{T}$ is bounded and that a functional $f$ in $X^{*}$ annihilates $\operatorname{Ran}(A-i \mu)+\operatorname{ker}(A-i \mu)$. For any $x$ in $X$, le ${ }^{+}$

$$
x_{n}=\frac{1}{n} \int_{0}^{n} e^{-i \mu t} T(t) x d t,
$$

and let $y$ be any weak ${ }^{*}$ limit point of $\left(x_{n}\right)$ in $X^{\odot *}$. Then $T^{\odot *}(t) y=e^{i \mu t} y$, so $y \in X^{\odot \odot}$. Thus if $\mathscr{T}$ is sun-reflexive, or if $\{T(t) x: t \geq 0\}$ is relatively weakly compact, then $y \in X$, so $y \in \operatorname{ker}(A-i \mu)$, and $f(y)=0$. But $f(x)=$ $f(y)=0$, since $T^{*}(t) f=e^{i \mu t} f$. Thus any bounded semigroup, which is either sun-reflexive or weakly almost periodic-in particular, any bounded semigroup on a reflexive space-is almost periodic, provided that $\sigma(A) \cap i \mathbf{R}$ is countable.

\section{Stabilization of contraction semigroups on Hilbert space}

Let $\mathscr{T}$ be a $C_{0}$-semigroup of contractions on a Hilbert space $X$, with generator $A$. Let $X_{u}(\mathscr{T})$ be the unitary subspace of $\mathscr{T}$, so that

$$
X_{u}(\mathscr{T})=\left\{x \in X:\|T(t) x\|=\|x\|=\left\|T(t)^{*} x\right\| \text { for all } t>0\right\} .
$$

Then $X_{u}(\mathscr{T})$ is a reducing subspace for $\mathscr{T}, \mathscr{T} \mid X_{u}(\mathscr{T})$ is a unitary semigroup on $X_{u}(\mathscr{T})$, and $T(t) x \rightarrow 0, T(t)^{*} x \rightarrow 0$ weakly as $t \rightarrow \infty$, for any $x$ in $X_{u}(\mathscr{T})^{\perp}$. If $\sigma(A) \cap i \mathbf{R}$ is null, then $\|T(t) x\| \rightarrow 0$ and $\left\|T(t)^{*} x\right\| \rightarrow 0$ for all $x$ in $X_{u}(\mathscr{T})^{\perp}$ [15]. If $X_{u}(\mathscr{T})=\{0\}$, then $\mathscr{T}$ is said to be completely nonunitary.

Let $Y$ be another Hilbert space, and $B: Y \rightarrow X$ be a bounded linear operator. For any bounded linear operator $K: X \rightarrow Y, A+B K$ generates a $C_{0}$-semigroup $\mathscr{S}_{K}=\left\{S_{K}(t): t \geq 0\right\}$ on $X$. If $S_{K}(t) x \rightarrow 0$ in norm (respectively, weakly) as $t \rightarrow \infty$ for all $x$ in $X$, then $(A, B)$ is said to be strongly (resp., weakly) stabilizable, and $K$ is said to be a stabilizer of $(A, B)$. We shall be particularly interested in the case when $K=-B^{*}$, so that $\mathscr{S}_{K}$ is a contraction semigroup, which we shall denote simply by $\mathscr{S}$.

The problem of stabilizability has been considered by several authors $[20,16$, 12,10 ] with applications to control theory in mind. Using almost periodicity, we shall give a proof of a slightly improved version of an elegant result obtained in $[10]$.

Lemma $10[16,12]$. Let $\mathscr{S}$ be the semigroup generated by $A-B B^{*}$. Then $X_{u}(\mathscr{S}) \subseteq X_{u}(\mathscr{T}) \cap \operatorname{ker} B^{*}$. Moreover, $X_{u}(\mathscr{S})$ is a reducing subspace for $\mathscr{T}$ and $T(t) x=S(t) x \quad\left(x \in X_{u}(\mathscr{P}), t \geq 0\right)$. 
Proof. For $x$ in $X_{u}(\mathscr{S}) \cap D(A)$,

$$
0=\operatorname{Re}\left(\left(A-B B^{*}\right) S(t) x, S(t) x\right) \leq-\left\|B^{*} S(t) x\right\|^{2} .
$$

Hence $B^{*} S(t) x=0$. Now

$$
T(t) x=\lim _{n \rightarrow \infty}\left[S(t / n) e^{(t / n) B B^{*}}\right]^{n} x=S(t) x .
$$

Similarly, $T(t)^{*} x=S(t)^{*} x$ for $x$ in $X_{u}(\mathscr{S}) \cap D\left(A^{*}\right)=X_{u}(\mathscr{S}) \cap D(A)$, and all the results follow.

Let $M_{c}$ be the closed linear span of $\{T(t) B y: y \in Y, t \geq 0\}$, and $M_{c}^{*}$ be the closed linear span of $\left\{T(t)^{*} B y: y \in Y, t \geq 0\right\}$. Korobov and Sklyar [10] proved variants of the following three lemmas.

Lemma 11. Let $\mathscr{S}$ be the semigroup generated by $A-B B^{*}$. Then $X_{u}(\mathscr{S}) \subseteq$ $X_{u}(\mathscr{T}) \cap M_{c}^{\perp} \cap M_{c}^{* \perp}$. If $i \mathbf{R}$ is not contained in $\sigma(A)$, then $X_{u}(\mathscr{S})=X_{u}(\mathscr{T}) \cap$ $M_{c}^{\perp}=X_{u}(\mathscr{T}) \cap M_{c}^{* \perp}$. In particular in this case, $\mathscr{S}$ is completely nonunitary if and only if $X_{u}(\mathscr{T}) \cap M_{c}^{\perp}=\{0\}$.

Proof. Let $x \in X_{u}(\mathscr{S})$. By Lemma 10, $x \in X_{u}(\mathscr{T})$ and $B^{*} T(t) x=B^{*} T(t)^{*} x$ $=0$ for all $t \geq 0$, so $x \in M_{c}^{\perp} \cap M_{c}^{* \perp}$.

Conversely, suppose that $x \in N:=X_{u}(\mathscr{T}) \cap M_{c}^{* \perp}$. Then $B^{*} T(t) x=0$ for all $t \geq 0$, and

$$
S(t) x=\lim _{n \rightarrow \infty}\left[e^{-(t / n) B B^{*}} T(t / n)\right]^{n} x=T(t) x .
$$

Moreover, $N$ is $\mathscr{T}$-invariant and $T(t) \mid N$ is isometric. Since $\sigma(A \mid N) \cap i \mathbf{R} \subseteq$ $A \sigma(A \mid N) \cap i \mathbf{R} \subseteq \sigma(A) \cap i \mathbf{R}$, if $i \mu \notin \sigma(A)$, then $i \mu \notin \sigma(A \mid N)$. If $i \mathbf{R}$ is not contained in $\sigma(A)$, it follows that $\mathscr{S}|N=\mathscr{T}| N$ extends to a unitary group on $N$ (see, for example, [13]). Thus $N \subseteq X_{u}(\mathscr{S})$. Similarly, $X_{u}(\mathscr{T}) \cap M_{c}^{\perp} \subseteq$ $X_{u}(\mathscr{S})$ if $i \mathbf{R}$ is not contained in $\sigma(A)$.

Lemma 12. Let $A$ and $B$ be as above. Then

$$
\sigma\left(A-B B^{*}\right) \cap i \mathbf{R} \subseteq \sigma(A) \cap i \mathbf{R}, \quad P \sigma\left(A-B B^{*}\right) \cap i \mathbf{R} \subseteq P \sigma(A) \cap i \mathbf{R} .
$$

Moreover, if $\left(A-B B^{*}\right) x=i \mu x$ for some $x$ in $D(A)$ and some real $\mu$, then $A x=i \mu x$ and $B^{*} x=0$.

Proof. Let $i \mu \in \sigma\left(A-B B^{*}\right) \cap i \mathbf{R}$. Then $i \mu \in \partial \sigma\left(A-B B^{*}\right) \subseteq A \sigma\left(A-B B^{*}\right)$, so there is a sequence $\left(x_{n}\right)$ in $X$ such that $\left\|x_{n}\right\|=1$ and $\left\|\left(A-B B^{*}-i \mu\right) x_{n}\right\| \rightarrow 0$. Then

$$
0 \geq-\left\|B^{*} x_{n}\right\|^{2} \geq \operatorname{Re}\left(\left(A-B B^{*}-i \mu\right) x_{n}, x_{n}\right) \rightarrow 0,
$$

so $\left\|B^{*} x_{n}\right\| \rightarrow 0$ and $\left\|(A-i \mu) x_{n}\right\| \rightarrow 0$. Thus $i \mu \in \sigma(A)$. The other statement is proved similarly.

If $\mathscr{T}$ is an almost periodic contraction semigroup on a Hilbert space $X$, then (in the notation of $\S 4), X_{b}(\mathscr{T})=X_{u}(\mathscr{T})$.

Lemma 13. If $X_{u}(\mathscr{T}) \cap M_{c}^{\perp} \cap M_{c}^{* \perp}=\{0\}$, then $(A, B)$ is weakly stabilizable, and $-B^{*}$ acts as stabilizer. Conversely, if $\mathscr{T}$ is almost periodic, and $(A, B)$ is weakly stabilizable, then $X_{u}(\mathscr{T}) \cap M_{c}^{\perp}=\{0\}$, and $-B^{*}$ acts as stabilizer. 
Proof. The first statement follows from Lemma 11, which shows that $\mathscr{S}$ is completely nonunitary, hence weakly stable.

Now suppose that $(A, B)$ is weakly stabilized by $K$, and let $x \in M_{c}^{\perp}$. For any $t \geq 0$ and $y$ in $Y,(x, T(t) B y)=0$, so $\left(x, T(t) e^{s B K} z\right)=(x, T(t) z)$ for all $z$ in $X, s \geq 0$. Hence

$$
\left(x,\left(T(t / n) e^{(t / n) B K}\right)^{n} z\right)=(x, T(t) z) .
$$

Letting $n \rightarrow \infty$,

$$
\left(x, S_{K}(t) z\right)=(x, T(t) z) .
$$

It follows that $T(t)^{*} x \rightarrow 0$ weakly as $t \rightarrow \infty$. Hence $x \in X_{b}(\mathscr{T})^{\perp}=X_{s}(\mathscr{T})$. Thus $M_{c}^{\perp} \subseteq X_{s}(\mathscr{T}), X_{u}(\mathscr{T}) \cap M_{c}^{\perp}=\{0\}$, and $X_{u}(\mathscr{S})=\{0\}$ by Lemma 11 . Hence $\mathscr{S}$ is weakly stable.

Korobov and Sklyar proved that conditions (1), (3), (4), and (5) of Theorem 14 are equivalent under the assumption that $\sigma(T(1)) \cap \Gamma$ is countable. If $\sigma(T(1)) \cap \Gamma$ is null, they proved that (1), (3), and (4) are equivalent. Since $\sigma(T(1))$ contains $\left\{e^{\lambda}: \lambda \in \sigma(A)\right\}$, this latter condition is stronger than $\sigma(A) \cap$ $i \mathbf{R}$ being countable. Similarly, using [15, p. 149] one may prove that (1), (3), and (4) are equivalent if $\sigma(A) \cap i \mathbf{R}$ is null.

Theorem 14. Let $\mathscr{T}=\{T(t): t \geq 0\}$ be a $C_{0}$-semigroup of contractions on a Hilbert space $X$, with generator $A$, and let $B$ be a bounded linear operator from a Hilbert space $Y$ into $X$. Suppose that $\sigma(A) \cap i \mathbf{R}$ is countable. Then the following are equivalent:

(1) $(A, B)$ is strongly stabilizable,

(2) $(A, B)$ is weakly stabilizable,

(3) $X_{u}(\mathscr{T}) \cap M_{c}^{\perp}=\{0\}$,

(4) $X_{u}(\mathscr{T}) \cap M_{c}^{* \perp}=\{0\}$,

(5) there do not exist real $\mu$ and $x$ in $\operatorname{ker} B^{*}, x \neq 0$, such that $A x=i \mu x$. Under these conditions, $-B^{*}$ acts as stabilizer.

Proof. Note first that $\mathscr{T}$ is almost periodic (see the remark after Theorem 8). Moreover, by Lemma 12, $\sigma\left(A-B B^{*}\right) \cap i \mathbf{R}$ is also countable, so $\mathscr{S}$ is also almost periodic.

(1) $\Rightarrow(2)$. This is trivial.

$(2) \Rightarrow(3)$ and $(4)$. Since $\mathscr{T}$ is almost periodic, this follows from Lemmas 13 and 11 .

(3) or $(4) \Rightarrow(1)$. By Lemma $13, \mathscr{P}$ is weakly stable. Since $\mathscr{S}$ is almost periodic, it follows that $\mathscr{P}$ is strongly stable.

(3) $\Rightarrow(5)$. If $A x=i \mu x$ and $B^{*} x=0$, then $T(t) x=e^{i \mu t} x, T(t)^{*} x=$ $e^{-i \mu t} x, B^{*} T(t)^{*} x=0$, so $x \in X_{u}(\mathscr{T}) \cap M_{c}^{\perp}$.

(5) $\Rightarrow$ (1). It follows from Lemma 12 that $A-B B^{*}$ has no purely imaginary eigenvalues. Since $\mathscr{P}$ is almost periodic, it follows from the splitting theorem that $\mathscr{S}$ is strongly stable. 
A $C_{0}$-semigroup $\mathscr{T}$ is quasi-compact if, for some $t>0, T(t)=K+V$, where $K$ is compact and $\|V\|<1$. The following version of the YosidaKakutani Mean Ergodic Theorem [21] is well known.

Theorem 15. Let $\mathscr{T}=\{T(t): t \geq 0\}$ be a bounded quasi-compact $C_{0}$-semigroup on a Banach space $X$. Then $\mathscr{T}$ is almost periodic, $X_{b}(\mathscr{T})$ is finite dimensional, and $\left\|T(t) \mid X_{s}(\mathscr{T})\right\| \rightarrow 0$ as $t \rightarrow \infty$.

In particular, if $A$ generates a bounded quasi-compact semigroup, then $\sigma(A)$ $\cap i \mathbf{R}$ is finite, and we obtain the following corollary of Theorem 14. In the case when $B$ is compact, this result was obtained by O'Brien [16], who showed moreover that the five conditions are equivalent to exponential stability of $\mathscr{S}$ $(\|S(t)\| \rightarrow 0$ as $t \rightarrow \infty)$.

Corollary 16. Let $\mathscr{T}=\{T(t): t \geq 0\}$ be a bounded quasi-compact $C_{0}$-semigroup on a Hilbert space $X$, with generator $A$, and let $B$ be a bounded linear operator from a Hilbert space $Y$ into $X$. Then the five conditions of Theorem 14 are equivalent. Moreover, under those conditions, $-B^{*}$ acts as a stabilizer.

Remark. Quasi-compact semigroups are examples of the compactifying semigroups introduced in $[17,18]$. A semigroup $\mathscr{T}$ is said to be compactifying if there is a compact subset $\Omega$ of $X$ such that $d(T(t) x, \Omega) \rightarrow 0$ as $t \rightarrow \infty$, whenever $\|x\| \leq 1$ (where $d$ denotes metric distance in $X$ ). It was proved in [18] that $\mathscr{T}$ is compactifying if and only if $\mathscr{T}$ is almost periodic and $X_{b}(\mathscr{T})$ is finite dimensional.

\section{Single OPERATORS}

Let $T$ be a bounded linear operator on a Banach space $X$. There are analogues of the results of $\S 2$ for the discrete semigroup $\left\{T^{n}: n \geq 0\right\}$, but they are of relatively limited interest. For example, the analogue of Theorem 1 has another elementary proof. For, if $\sigma(T) \cap \Gamma$ is empty, where $\Gamma=\{\lambda \in \mathbf{C}:|\lambda|=1\}$, then there is a spectral decomposition $X=X_{1} \oplus X_{2}, T=T_{1} \oplus T_{2}$, where $T_{2}$ is invertible and $T_{1}$ and $T_{2}^{-1}$ have spectral radii less than 1 . Thus if $x \in X$ and $\left\|T^{n} x\right\|$ is bounded (or if $\left\|T^{n} x\right\|=o\left(a^{n}\right)$ for all $a>1$ ), then $x \in X_{1}$ and $\left\|T^{n} x\right\|=\left\|T_{1}^{n} x\right\| \rightarrow 0$.

There is an analogue of Theorem 2 and Corollary 3. If $\sigma(T) \cap \Gamma$ is countable, $R \sigma\left(T^{*}\right) \cap \Gamma$ is empty, and $\left\|T^{n} x\right\|$ is bounded, then $T^{n} x \rightarrow 0$ weakly. One might expect this result to remain true if it is assumed only that $\left\|T^{n} x\right\|=o\left(a^{n}\right)$ for all $a>1$. However, this is false, as may be seen by taking $X=l^{2}$ and $T=I+S$, where

$$
S x=\left(0, \frac{x_{1}}{2}, \frac{x_{2}}{3}, \frac{x_{3}}{4}, \ldots\right) .
$$

Then $\left\|T^{n}\right\|=o\left(a^{n}\right)$ for all $a>1, \cdot \sigma(T)=\{1\}, 1 \notin P \sigma(T)=R \sigma\left(T^{*}\right)$. But $\left\|T^{n}\right\| \rightarrow \infty$, and there exists $x$ such that $\left\|T^{n} x\right\|$ is unbounded.

There is also an analogue of Theorem 8. If $\left\{T^{n}\right\}$ is bounded, $\sigma(T) \cap \Gamma$ is countable, and $\operatorname{Ran}(T-\lambda)+\operatorname{ker}(T-\lambda)$ is dense in $X$ for each $\lambda$ in $\Gamma$, then $\left\{T^{n} x: n \geq 1\right\}$ is relatively compact, for each $x$ in $X$. 
Taking the discrete semigroup $\{T(n): n \geq 1\}$ contained in Example 7 provides counterexamples to other possible conjectures.

\section{REFERENCES}

1. W. Arendt and C. J. K. Batty, Tauberian theorems and stability of one-parameter semigroups, Trans. Amer. Math. Soc. 306 (1988), 837-852.

2. C. J. K. Batty, Tauberian theorems for the Laplace-Stieltjes transform, Trans. Amer. Math. Soc. 322 (1990), 783-804.

3. Ph. Clément et al., One-parameter semigroups, North-Holland, Amsterdam, 1987.

4. K. de Leeuw and I. Glicksberg, Applications of almost periodic compactifications, Acta Math. 105 (1961), 63-97.

5. B. de Pagter, A characterization of sun-reflexivity, Math. Ann. 283 (1989), 511-518.

6. N. Dunford and J. T. Schwartz, Linear operators. I, Wiley, New York, 1958.

7. E. Hille and R. S. Phillips, Functional analysis and semigroups, Amer. Math. Soc., Providence R.I., 1957.

8. A. E. Ingham, On Wiener's method in Tauberian theorems, Proc. London Math. Soc. (2) 38 (1935), 458-480.

9. J. Korevaar, On Newman's quick way to the prime number theorem, Math. Intelligencer 4 (1982), 108-115.

10. V. I. Korobov and G. M. Sklyar, On the problem of strong stabilizability of contraction systems on Hilbert space, Differential Equations 20 (1984), 1320-1326.

11. U. Krengel, Ergodic theorems, De Gruyter, Berlin, 1985.

12. N. Levan and L. Rigby, Strong stabilizability of linear contractive control systems on Hilbert space, SIAM J. Control Optim. 17 (1979), 23-35.

13. Yu. I. Lyubich and Vũ Quôc Phóng, Asymptotic stability of linear differential equations in Banach spaces, Studia Math. 88 (1988), 37-42.

14. R. Nagel (ed.), One-parameter semigroups of positive operators, Lecture Notes in Math., vol. 1184, Springer, Berlin, 1986.

15. B. Sz.-Nagy and C. Foias, Harmonic analysis of operators on Hilbert space, North-Holland, Amsterdam, 1970.

16. R. E. O'Brien, Contraction semigroups, stabilization, and the mean ergodic theorem, Proc. Amer. Math. Soc. 71 (1978), 89-94.

17. Vũ Quôc Phóng, Représentations compactifiantes de semi-groupes, C.R. Acad. Sci. Paris Sér. A 305 (1987), 273-274.

18. _ Applications of Suskevic kernel to semigroup actions and representations, preprint, 1987.

19. Vũ Quôc Phóng and Yu. I. Lyubich, A spectral criterion for almost periodicity of oneparameter semigroups, Teor. Funktsii Funktsional. Anal. i Prilozhen. 47 (1987), 36-41. (Russian).

20. M. Slemrod, A note on complete controllability and stabilizability of linear control systems in Hilbert space, SIAM J. Control Optim. 12 (1974), 500-508.

21. K. Yosida and S. Kakutani, Operator theoretical treatment of Markov processes and mean ergodic theorems, Ann. of Math. (2) 42 (1941), 188-228.

22. D. Zagier, Short proof of the prime number theorem, unpublished manuscript.

St. John's COllege, OXford OX1 3JP, ENGLAND

Institute of Mathematics, P.O. Box 631, 10000 Hanoi, Vietnam 